\title{
Effekte der intravenösen Nikotinsäureinfusion auf Substrat-, Metabolit- und Hormonkonzentrationen im Blut beim juvenil-manifestierten Diabetes mellitus
}

\author{
D. Michaelis, H.-J. Hahn, R. Michael, S. Knospe, H. Schäfer, E. Jutzi und P. Wulfert
}

\begin{abstract}
Institut für Diabetes "Gerhardt Katsch" - Forschung und Behandlung - Karlsburg und Garz, Bereich Klinik Karlsburg (Institutsdirektor und Leiter der Klinik: Prof. Dr. med. habil. H. Bibergeil) und Bereich experimentelle Diabetesforschung (damaliger Leiter: Doz. Dr. med. habil. H. G. Lippmann)
\end{abstract}

Eingegangen am 18. Dezember 1969

Effects of the intravenous infusion of nicotinic acid on blood concentrations of substrates, metabolites and hormones in juvenile-anset diabetes

Summary. The effect of a $30 \mathrm{~min}$ infusion of nicotinic acid on the blood levels of glucose, pyruvate, lactate, FFA, glycerol, catecholamines, and 11-OH-corticosteroids was investigated over $180 \mathrm{~min}$ in 73 insulin-dependent diabetics with different clinical states of metabolism and in 7 healthy subjects. The action of nicotinic acid on insulin secretion was measured in vivo in some diabetic and some healthy subjects by means of ILA. Studies on insulin secretion in vitro were done by measuring the insulin release from isolated islets of mouse pancreas. The insulin content of the incubation medium was estimated with the radio-immunological method. - It was shown that nicotinic acid in vitro stimulates insulin release from $\mathrm{B}$ cells significantly. In vivo the rise of serum ILA after nicotinic acid infusion was only detectable in healthy subjects and in diabetics of the stable type. Furthermore, a negative correlation was demonstrated between the peak of the FFA-rebound induced by nicotinic acid and the stimulatory effect of the drug on insulin secretion. The behaviour of blood glucose during infusion of nicotinic acid together with the daily insulin dosage permitted a metabolic distinction to be made between diabetics of the stable and unstable ('brittle') type.

Effets de l'infusion d'acide nicotinique par voie veineuse sur la concentration des substrats, métabolites et hormones du sang dans le diabète qui se manifeste à l'âge juvénile

Résumé. Chez 73 diabétiques traités par l'insuline qui montrent un comportement clinique différent du métabolisme, et chez 7 sujets normaux, nous avons fait une épreuve de charge en acide nicotinique par voie veineuse pendant $30 \mathrm{~min}$ et nous avons analysé les taux sanguins du glucose, du pyruvate, du lactate, des FF A, du glycérol, des catécholamines et de 11-OH-CS pendant une période de $180 \mathrm{~min}$. En outre nous avons mesuré l'influence de l'acide nicotinique sur l'insulino-sécrétion, in vivo chez quelques sujets diabétiques et normaux par la méthode radiobiologique (ILA), in vitro sur des îlots de Langerhans isolés de souris par la méthode radio-immunologique (IRI). In vitro nous avons vu une élévation significative de l'insulino-sécrétion, in vivo on peut montrer une élévation de l'TLA seulement chez les sujets normaux et chez les diabétiques ayant un métabolisme stable. L'élévation de "FFA-rebound" secondaire provoquée par l'infusion de l'acide nicotinique était en corrélation négative avec l'effet stimulant sur l'insulino-sécrétion. Ce comportement permet une différentiation métabolique des diabétiques de type instable et stable en tenant compte de la glycémie et de la dose quotidienne d'insuline nécessaire pour la compensation du métabolisme.

Zusammenfassung. Bei 73 insulinbedürftigen Diabetikern mit klinisch differentem Stoffwechselverhalten und 7 Gesunden wurden eine 30-minütige Nicotinsäureinfusion durchgeführt und die Blutparameter von Glucose, Pyruvat, Lactat, FFS, Glycerin, Katecholaminen und 11-OH-CS über einen Zeitraum von $180 \mathrm{~min}$ analysiert. Außerdem wurde die Insulinsekretion in vivo bei einigen diabetischen und gesunden Probanden mittels der ILA, in vitro an isolierten Langerhans'schen Inseln aus Mäusepankreata mittels dem IRI unter Nikotinsäureeinwirkung untersucht. Es konnte demonstriert werden. daß Nikotinsäure in vitro zu einer significanten Steigerung der Insulinausschüttung führt. In vivo ist eine Stimulation der ILA nur bei Gesunden und stoffwechselstabilen Diabetikern nachweisbar. Das Ausmaß des sekundären Nicotinsäureinduzierten FFS-Rebound steht in negativer Relation zur Stimulierbarkeit der ILA. Es gestattet unter Einbeziehung des Blutglucoseverhaltens und des exogenen, zur Stoffwechselkompensation erforderlichen täglichen Insulinbedarfs eine metabolische Differenzierung des stoffwechsellabilen und stoffwechselstabilen Diabetestyps.

Key-words: Nicotinic acid infusion, FFA-rebound, stable and brittle diabetes, insulin secretory response.
Der juvenil-manifestierte, insulinbedürftige Diabetes mellitus wird im internationalen Schrifttum häufig mit dem "juvenile type" Diabetes gleichgesetzt, was jedoch keinesfalls berechtigt ist. Allein das differente Stoffwechselverhalten auch das insulinabhängigen Diabetes, welches im klinischen Sprachgebrauch zur Unterscheidung zwischen dem stabilen (stable) und labilen (instable, brittle) (Woodyatt 1934) Stoffwechseltyp führte, spricht gegen einen einheitlichen Diabetestyp. Diese Differenzierung bezieht sich auf das klinische Erscheinungsbild, wobei die variable Insulinsensitivi- tät, die Ketose- und Hypoglykämieneignung mit nachfolgender Stoffwechseldekompensation (Molnar 1964) ohne erkennbare Ursachen, wie Variationen der Zusammensetzung und Absorption der Diät, Infectionen oder Alterationen der Leber sowie der Aktivität von Hypophyse, Schilddrüse und Nebennieren (Joslin et al. 1959) als Charakteristika des labilen Stoffwechseltyps gelten. Infolge Fehlens biochemischer und hormoneller Charakteristika ist diese klinische Unterteilung mit dem Mangel der Subjektivität behaftet. Dies gilt um so mehr, als die Symptomatik der Stoffwechsellabilität 
durch iatrogenen Hyperinsulinismus (Somogy 1961) respektive unkontrollierte Nahrungszufuhr erzengt werden kann.

Das absolute endogene Insulindefizit wird nach Vallance-Owen et al. (1955), Gepts (1965), Parker et al. (1968), als Kennzeichen des juvenil-manifestierten Diabetes angesehen. Jedoch spricht das differente Stoffwechselverhalten sowie das zunehmend häufigere Er. scheinen „milder" Diabetesformen in den juvenilen Altersklassen (Dolger 1961, Pildes u. Cornblath 1967, Fajans u. Conn 1965) gegen die generelle Annahme eines absoluten Insulinmangels beim juvenil-manifestierten Diabetes mellitus.

Die hohe Insulinsensitivität des Fettgewebes einerseits, der biphasische Wirkungsablauf der Nicotinsäure im Fettstoffwechsel (Carlson und Orö 1962, Pinter und Pattee 1967) andererseits, waren Veranlassung, diesen Lipolysehemmer im akuten Belastungsverfahren bei stoffwechselstabilen und stoffwechsellabilen Diabetikern einzusetzen. Dabei sollten kausale Beziehungen zwischen Metabolitverhalten und Hormonkonzentrationsänderungen im Blut aufgezeigt und Möglichkeiten der metabolischen Differenzierung beider Stoffwechseltypen geprüft werden.

\section{Methodik}

a) klinische Versuche. Die 30-minütige Na-Nicotinat-Infusion ${ }^{1}$ wurde bei 73 insulinbedürftigen Diabetikern und 7 Stoffwechselgesunden durchgeführt. Die Einteilung der Diabetiker in 3 Gruppen wurde nach den vorherrschenden klinischen Merkmalen vorgenommen, wobei die Einstellbarkeit des KH-Stoffwechsels, gemessen an den Fluktuationen der Glykämie und Glucosurie, der Ketose- und Hypoglykämieneigung, sowie die Symptomatik der vegetativen Labilität (Dermographismus, Lidflattern, Zungenwogen, feuchtkalte Hände) als Kardinalsymptome galten. 17 stoffwechselstabile Diabetiker (Gruppe I) waren durch ein mittleres Alter von 24,9 Jahren, einer Diabetesdauer von 5,0 Jahren, einem exogenen Insulinbedarf von $33,5 \pm 1,51 \mathrm{E} /$ die bei einer Zufuhr von 301,2 g Kohlenhydraten/die charakterisiert. Das Durchschnittsalter der 16 stoffwechselstabilen Diabetiker mit vegetativer Labilität (Gruppe II) betrug 27,2 Jahre, die Diabetesdaver 4,8 Jahre und der Insulinbedarf $30,0 \pm 2,83$ $\mathrm{E} /$ die bei einer Zufuhr von $266,4 \mathrm{~g}$ Kohlenhydraten/die. 40 stoffwechsellabile Diabetiker (Gruppe III) waren durch ein mittleres Alter von 29,1 Jahren, eine Diabetesdauer von 9,2 Jahren, einen exogenen Insulinbedarf von $55,2 \pm 1,81 \mathrm{E} /$ die bei einer Zufuhr von $297,6 \mathrm{~g}$ Kohlenhydraten/die gekennzeichnet. Das Durchschnittsalter der gesunden Probanden (Gruppe IV) betrug 25,4 Jahre.

Nach dem nächtlichen, 12-stündigen Fasten wurde den Probanden um 6.30 Uhr eine Kanüle in die Cubitalvene gelegt und eine $0,9 \%$-ige NaCL-Infusion zum

1 Nikodan ,Philopharm“.
Offenhalten der Kanüle angeschlossen. Nach Abnahme der Nüchternparameter um 7.00 Uhr wurden in den nächstfolgenden $30 \mathrm{~min} 300 \mathrm{mg}$ Na-Nicotinat, gelöst in $150 \mathrm{ml}$ physiologischer Na-CL-Lösung, infundiert. Die Bestimmung der kapillären Blutglucose nach Hugget u. Nixon (1957) sowie der freien Fettsäuren (FFS) nach Duncombe (1964) erfolgte in 15- bzw. 30-minütigen Intervallen. Die Analysen von Glycerin nach Eggstein u. Kreutz (1966a, b), Aceton nach Krainick (1938), Pyruvat und Lactat in Anlehnung an Hohorst et al. (1959) und Bücher et al. (1962) mittels des optischen Tests, der Plasmakatecholamine nach Anton u. Sayre (1962), der 11-Hydroxycorticoide (11-OH-CS) nach einer auf Grund der Angaben von Nielsen u. Asfeldt (1967) modifizierten Methode sowie der fat-pad ILA

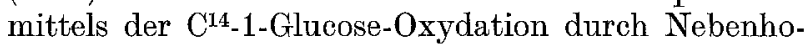
denfettgewebe von Ratten nach Renold et al. (1960) wurden zu den beobachteten maximalen Konzentrationsänderungen der FFS, nämlich zu den Blutentnahmezeiten $0,45,90$ und $150 \mathrm{~min}$, vorgenommen.

b) in vitro-Versuche. Die Incubationsversuche mit $1 \mathrm{mM}$ Na-Nicotinat wurden an Langerhans'schen Inseln aus Pankreata normaler weißer Mäuse (Stamm AB, 26-30 g, Standardfutter - VEB Mischfutterwerk Altglienicke - ad libitum) durchgeführt. Die Isolierung erfolgte durch kurze Kollagenasevorbehandlung (Biochemical Corporation, Worthington) mit anschlieBender Feinpräparation unter dem Stereomikroskop in in einer peltiergekühlten Kammer bei $0^{\circ} \mathrm{C}$. Die Tiere fasteten 16 Std. vor dem Tod, erhielten lediglich Wasser ad libitum. Die Incubation von 10 isolierten Inseln erfolgte in $1 \mathrm{ml}$ Krebs-Ringer-Bicarbonatpuffer $(\mathrm{pH}$ 7,$4 ; 2 \mathrm{mg} / \mathrm{ml}$ Glucose und lyophilisiertes Rinderalbu$\min$ ) bei $37^{\circ} \mathrm{C} 120 \mathrm{~min}$ mit einer Schüttelfrequenz von $80 \mathrm{~min}^{-1}$ (Gasphase 95\% $\mathrm{O}_{2}$ und $5 \% \mathrm{CO}_{2}$ ). Die Versuche wurden im Paarvergleich durchgeführt, d.h., daß aus einem Pankreas die Inseln für die Na-Nicotinat und Kontrollincubation gewonnen wurden. Das Insulin wurde aus $1: 20$ verdünntem Medium radioimmunologisch (IRI) mit der Doppelantikörpermethode (Hales u. Randle 1963) unter Verwendung von $\mathbf{J}^{125}$-Insulin (Greenwood et al. 1963) gegen Rinderinsulin als Standard gemessen. Die Insulinsekretionsrate wurde aus der Differenz zwischen dem vor Incubationsbeginn erhaltenen Nullwert und dem nach 120 min Incubation gemessenen Wert berechnet und auf $10 \mu \mathrm{g}$ Gewebsprotein (Lowry et al. 1951) normiert.

Zur statistischen Charakterisierung errechneten wir die Mittelwerte und deren mittlere Fehler(S.E.M.). Die Beurteilung der Differenzen zwischen den Mittelwerten erfolgte nach dem t-Test. Bei der rechnerischen Differenzierung der Stoffwechseltypen wurde die lineare Diskriminanzanalyse (Weber 1964) angewandt.

\section{Ergebnisse}

\section{Fettstoffwechselparameter (Abb.1)}

a) FFS. Unter der 30-minütigen Nicotinsäureinfusion sinkt die FFS-Konzentration bei allen Proban- 
den bereits nach 15 min ab. Das Maximum der absoluten Konzentrationsverminderung wird nach $45 \mathrm{~min}$ beobachtet und steht in positiver Korrelation zum Ausgangswert (Gr. I: $r=0,942$; Gr. II: $r=0,943$; Gr. III: $r=0,872$; Gr. IV : $r=0,976 ; P<1 \%$ ).

Der FES-Rebound setzt bei stoffwechsellabilen Diabetikern bereits zwischen der 45 . und 60 . min ein $(+$ $0,065 \pm 0,025 \mathrm{mval} / \mathrm{l}, P<5 \%$ ). In den beiden Gruppen der stoffwechselstabilen Diabetiker läßt sich die beobachtete Konzentrationsänderung in diesem Zeitintervall mit $+0,018 \pm 0,037 \mathrm{mval} / \mathrm{I}(\mathrm{Gr} . \mathrm{I})$ und $+0,034$ $\pm 0,035 \mathrm{mval} / \mathrm{l}$ (Gr. II) statistisch nicht sichern. Das Maximum wird bei allen Probanden nach 150 min erreicht. Das Ausmaß des FFS-Rebound, gemessen zwi-
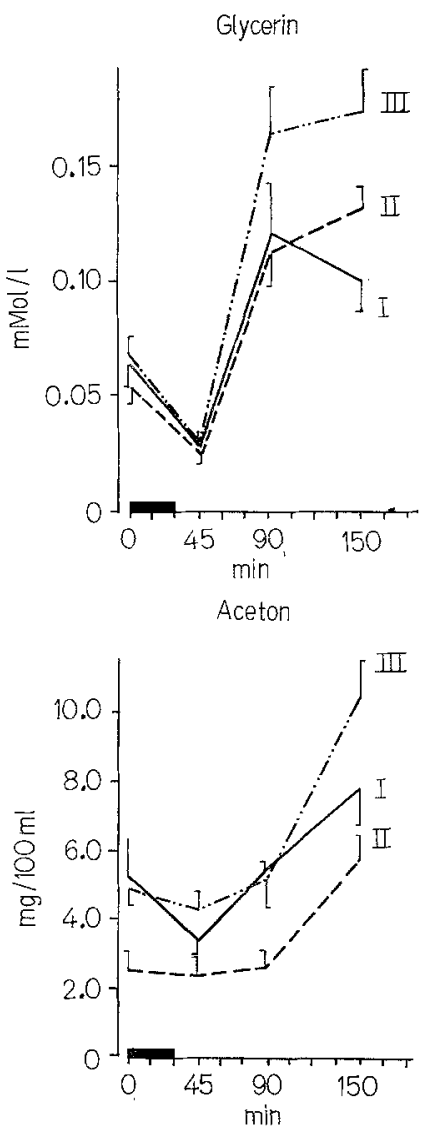

b) Glycerin. Als Ausdruck des Lipolyse-inhibierenden Effektes der Nicotinsäure tritt in den ersten $45 \mathrm{~min}$ des Versuchs ein Abfall der Glycerinkonzentration bei Diabetikern ein, dessen Ausmaß in signifikanter Korrelation zum Nüchternwert steht (Gr. I: $r=0,929$; Gr. II : $r=0,642 ;$ Gr. III $: r=0,942$ ). Nachfolgend setzt der Wiederanstieg ein, der in der Gruppe I zum Zeitpunkt $90 \mathrm{~min}$, in den Gruppen II und III zum Zeitpunkt 150 min das Maximum erreicht. Entsprechend den höheren FFS-Konzentrationen ist der Glycerin. Rebound im Zeitintervall 45 bis $150 \mathrm{~min}$ mit $0,106 \pm$ $0,007 \mathrm{mMol} / 1$ (Gr. II) bzw. 0,145 $\pm 0,017 \mathrm{mMol} / 1$ (Gr. III) im Vergleich zu stoffwechselstabilen Probanden $(0,073 \pm 0,016 \mathrm{mMol} / 1)$ stärker ausgeprägt $(1 \%<P$
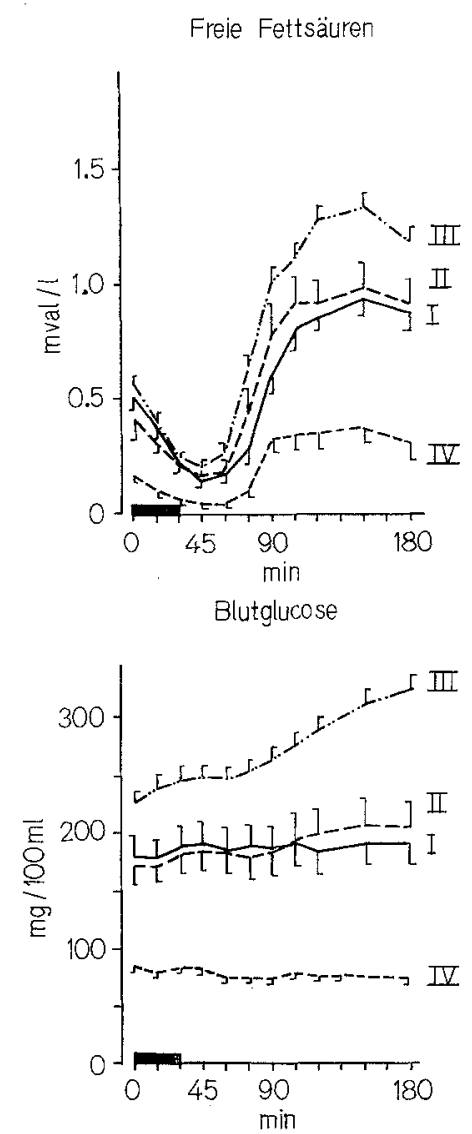

Abb. 1. Veränderungen der Blutkonzentrationen von Glucose und Fettstoffwechselparametern bei Diabetikern ( $\mathrm{I}=$ stw.-stabil, II = stw.-stabil + vegetativ-labil, III = stw.-labil) und Gesunden (IV) nach 30-minütiger Nicotinsäureinfusion

schen der 45. und 150. min, steigt in der Reihenfolge Stoffwechselgesunde $(+0,33 \pm 0,076 \mathrm{mval} / \mathrm{l})$, stoffwechselstabile Diabetiker $(+0,77 \pm 0,067 \mathrm{mval} / \mathrm{l})$, stoffwechselstabile Diabetiker mit vegetativer Labilität $(+0,83 \pm 0,094 \mathrm{mval} / 1)$, stoffwechsellabile Diabetiker $(+1,13 \pm 0,054 \mathrm{mval} / \mathrm{l})$ an. Dabei ist bemerkenswert, daß weder bei Gesunden noch bei den Diabetikern positive Korrelationen zwischen der Größenordnung des FFS-Rebound und dem Nüchternwert oder dem initialen FFS-Konzentrationsabfall bestehen.
$<5 \%$ ). Das Fehlen echter Korrelationen zwischen dem initialen Glycerinabfall und dem nachfolgenden Anstieg deutet an, daß der Rebound nicht als einfacher autoregulativer Vorgang anzusehen ist.

c) Aceton. Entsprechend der kurzfristigen Lipolysehemmung werden die Blutacetonkonzentrationen bei Diabetikern bis $45 \mathrm{~min}$ nach Versuchsbeginn nicht significant alteriert. Dagegen tritt in Relation zum beobachteten FFS-Rebound zwischen der 90. und 150. min eine Erhöhung ein, die sich bei Diabetikern der 
Gruppe II mit $3,19 \pm 1,20 \mathrm{mg} \%(P<5 \%)$ und der Gruppe III $(5,30 \pm 1,07 \mathrm{mg} \% ; P<1 \%)$ statistisch sichern läßt.

\section{Parameter des Kohlenhydratstoffwechsels}

a) Blutglucose (Abb.1). Die Blutglucosespiegel werden bei Gesunden und stoffwechselstabilen Diabetikern wie bei den NaCL-Kontrollversuchen weder während b) Pyruvat und Lactat (Tabelle 1). Die Konzentrationen beider Metaboliten werden durch die Nicotinsäureinfusion bei Diabetikern nicht beeinflußt. Der initial in den beiden stoffwechselstabilen Gruppen zu beobachtende Konzentrationsanstieg des Pyruvats bzw. der bei stoffwechsellabilen Diabetikern auftretende Abfall der Lactatkonzentration läßt sich statistisch nicht sichern.

Tabelle 1. Verhalten der Serumkonzentrationen von Pyruvat und Lactat bei Diabetikern unter Nicotinsäureinfusion (Mittelwerte $\pm S . E . M$.

\begin{tabular}{|c|c|c|c|c|c|c|c|c|c|}
\hline \multirow{2}{*}{$\begin{array}{l}\text { Stoff- } \\
\text { wechseltyp }\end{array}$} & \multirow[b]{2}{*}{$n$} & \multicolumn{4}{|c|}{ Pyruvat $\mu \mathrm{Mol} / \mathrm{g}$ Vollblut } & \multicolumn{4}{|c|}{ Lactat $\mu \mathrm{Mol} / \mathrm{g}$ Vollblut } \\
\hline & & $0^{\prime}$ & $45^{\prime}$ & $90^{\prime}$ & $150^{\prime}$ & $0^{\prime}$ & $45^{\prime}$ & $90^{\prime}$ & $150^{\prime}$ \\
\hline $\mathrm{I}$ & 10 & 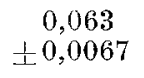 & $\begin{array}{c}0,076 \\
\pm 0,0062\end{array}$ & $\begin{array}{c}0,076 \\
\pm 0,0087\end{array}$ & $\begin{array}{c}0,064 \\
\pm 0,0095\end{array}$ & $\begin{array}{c}0,71 \\
\pm 0,047\end{array}$ & $\begin{array}{c}0,75 \\
+0,074\end{array}$ & $\begin{array}{c}0,93 \\
+0,220\end{array}$ & $\begin{array}{c}0,72 \\
\pm 0,073\end{array}$ \\
\hline II & 7 & $\begin{array}{c}0,069 \\
\pm 0,0112\end{array}$ & $\begin{array}{c}0,071 \\
\pm 0,0083\end{array}$ & $\begin{array}{c}0,060 \\
\pm 0,0084\end{array}$ & $\begin{array}{c}0,055 \\
\pm 0,0054\end{array}$ & $\begin{array}{c}0,73 \\
\pm 0,098\end{array}$ & $\begin{array}{c}0,69 \\
+0,102\end{array}$ & $\begin{array}{c}0,84 \\
\pm 0,255\end{array}$ & $\begin{array}{c}0,63 \\
\pm 0,044\end{array}$ \\
\hline III & 22 & $\begin{array}{c}0,070 \\
\pm 0,0047\end{array}$ & $\begin{array}{c}0,071 \\
\pm 0,0057\end{array}$ & $\begin{array}{c}0,065 \\
\pm 0,0052\end{array}$ & $\begin{array}{c}0,063 \\
\pm 0,0045\end{array}$ & $\begin{array}{c}0,85 \\
\pm 0,050\end{array}$ & $\begin{array}{c}0,72 \\
\pm 0,053\end{array}$ & $\begin{array}{c}0,78 \\
\pm 0,051\end{array}$ & $\begin{array}{c}0,85 \\
+0,068\end{array}$ \\
\hline
\end{tabular}

Tabelle 2. Plasmakatecholamin- und 11-OH-CS-Konzentrationen bei Diabetikern unter Nicotinsäureinfusion (Mittelwerte \pm S.E.M.)

\begin{tabular}{|c|c|c|c|c|c|c|c|c|c|c|c|}
\hline \multirow{2}{*}{$\begin{array}{l}\text { Stoffwechsel- } \\
\text { typ }\end{array}$} & \multicolumn{7}{|c|}{ Katecholamine $\mu \mathrm{g} / \mathrm{l}$} & \multicolumn{4}{|c|}{$11-\mathrm{OH}-\mathrm{CS} \mu \mathrm{g} / 100 \mathrm{ml}$} \\
\hline & $\bar{n}$ & $0^{\prime}$ & $45^{\prime}$ & $90^{\prime}$ & & $150^{\prime}$ & $n$ & $0^{\prime}$ & $45^{\prime}$ & $90^{\prime}$ & $150^{\prime}$ \\
\hline I & 10 & $\begin{array}{c}12,4 \\
\vdots \quad 1,73\end{array}$ & $\begin{array}{c}15,6 \\
\pm \quad 2,74\end{array}$ & $\begin{array}{c}\mathbf{1 3 , 3} \\
\pm \quad 2,07\end{array}$ & \pm & $\begin{array}{l}14,0 \\
1,72\end{array}$ & 8 & $\begin{array}{r}21,3 \\
\pm \quad 4,33\end{array}$ & $\begin{array}{c}12,2 \\
+\quad 1,70\end{array}$ & 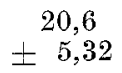 & $\begin{array}{r}13,3 \\
+\quad 2,31\end{array}$ \\
\hline II & 6 & $\begin{array}{ll} & 7,5 \\
\pm & 1,14\end{array}$ & $\begin{array}{l}\quad 9,1 \\
+\quad 2,36\end{array}$ & $\begin{array}{r}13,2 \\
+\quad 3,18\end{array}$ & \pm & $\begin{array}{l}9,3 \\
2,18\end{array}$ & 8 & $\begin{array}{c}15,4 \\
\pm \quad 4,30\end{array}$ & $\begin{array}{c}18,3 \\
+\quad 3,49\end{array}$ & $\begin{array}{c}10,5 \\
\pm \quad 3,37\end{array}$ & 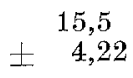 \\
\hline III & 21 & $\begin{array}{c}14,5 \\
\pm \quad 2,30\end{array}$ & $\begin{array}{c}14,8 \\
\pm \quad 2,22\end{array}$ & $\begin{array}{c}16,5 \\
\pm \quad 2,27\end{array}$ & \pm & $\begin{array}{c}15,4 \\
1,77\end{array}$ & 21 & $\begin{array}{c}17,8 \\
\pm \quad 2,03\end{array}$ & $\begin{array}{r}13,8 \\
\pm \quad 1,87\end{array}$ & $\begin{array}{c}17,2 \\
\pm \quad 2,04\end{array}$ & $\begin{array}{c}17,1 \\
+\quad 1,53\end{array}$ \\
\hline
\end{tabular}

Tabelle 3. Negative Relationen zwischen FFS-Rebound und Konzentrationsveränderungen der ILA unter Nicotinsäureinfusion

\begin{tabular}{|c|c|c|c|c|c|c|c|c|c|}
\hline \multirow[t]{2}{*}{ Stoffwechseltyp } & \multirow[t]{2}{*}{ Pat. Nr. } & \multirow{2}{*}{$\begin{array}{c}\text { Alter } \\
(\mathrm{J} .)\end{array}$} & \multirow{2}{*}{$\begin{array}{l}\text { DD } \\
(\mathbf{J} .)\end{array}$} & \multirow{2}{*}{$\begin{array}{l}\text { Insulinbe- } \\
\text { darf E/die }\end{array}$} & \multicolumn{4}{|c|}{ ILA $\mu \mathrm{E} / \mathrm{ml}$ Serum } & \multirow{2}{*}{$\begin{array}{l}\text { FFS-Anstieg } \\
45^{\prime}-150^{\prime} \\
\text { mval/1 }\end{array}$} \\
\hline & & & & & $0^{\prime}$ & $45^{\prime}$ & $90^{\prime}$ & $150^{\prime}$ & \\
\hline Stw.-labiler D. m. & $\begin{array}{r}15107 \\
5674 \\
3910\end{array}$ & $\begin{array}{l}32 \\
39 \\
42\end{array}$ & $\begin{array}{r}6 \\
12 \\
21\end{array}$ & $\begin{array}{l}40 \\
48 \\
54\end{array}$ & $\begin{array}{l}10 \\
10 \\
10\end{array}$ & $\begin{array}{l}34 \\
10 \\
17\end{array}$ & $\begin{array}{l}12 \\
10 \\
10\end{array}$ & $\begin{array}{l}10 \\
10 \\
10\end{array}$ & $\begin{array}{l}1,12 \\
2,00 \\
1,12\end{array}$ \\
\hline Stw.-stabiler D.m. & $\begin{array}{l}15036 \\
15154\end{array}$ & $\begin{array}{l}35 \\
31\end{array}$ & $\begin{array}{l}3 \\
2\end{array}$ & $\begin{array}{l}30 \\
44\end{array}$ & $\begin{array}{l}80 \\
20\end{array}$ & $\begin{array}{r}270 \\
90\end{array}$ & $\begin{array}{r}200 \\
50\end{array}$ & $\begin{array}{r}200 \\
32\end{array}$ & $\begin{array}{l}0,75 \\
0,58\end{array}$ \\
\hline Diätbedürftiger D. m. & 15011 & 31 & 0,5 & $\emptyset$ & 148 & 600 & 360 & 440 & 0,28 \\
\hline Stoffwechselgesunder & 13031 & 40 & $\emptyset$ & $\emptyset$ & 164 & 276 & 280 & 220 & 0,26 \\
\hline
\end{tabular}

der Nicotinsäureinfusion noch des nachfolgenden Untersuchungszeitraums signifikant verändert. Stoffwechsellabile Probanden zeichnen sich dagegen durch einen Konzentrationsanstieg der Glucose von $+98 \pm$ $8,97 \mathrm{mg} \%(P<1 \%)$ im Zeitintervall 0 bis $180 \mathrm{~min}$ aus. Dieser steht jedoch nicht in Zusammenhang mit der Nicotinsäureinfusion, da er auch in Kontrollversuchen mit physiologischer Kochsalzlösung bei stoffwechsellabilen Diabetikern in dieser Größenordnung $(+96,9 \pm$ $11,23 \mathrm{mg} \% ; P<1 \%$ ) auftrat.
3. Hormone (Tabelle 2)

a) Plasmakatecholamine. Bei Diabetikern der Gruppen I und III ist kein Nicotinsäureeffekt auf die Plasmakatecholaminkonzentration nachweisbar. Lediglich bei Probanden mit vegetativer Labilität, deren mittlere Nüchternwerte auffallend niedrig sind, wird eine ansteigende Tendenz bis zur 90 . min beobachtet, die sich statistisch jedoch nicht sichern läßt.

b) 11-OH-CS. Stoffwechsellabile (Gruppe III) und stoffwechselstabile Diabetiker (Gruppe I) reagieren un- 
ter Nicotinsäureeinwirkung initial mit einem Abfall der 11-OH-CS-Konzentration, die sich bei ersteren statistisch sichern läßt $(P<1 \%)$. Zwischen der 45 . und 90. min tritt in beiden Gruppen ein Wiederanstieg bis in Nïchternwerthöhe auf. Vegetativ-labile Probanden (Gruppe II) weisen ein konträres Verhalten der 11-OHCS-Konzentrationen auf. Der in den Gruppen I und III zu beobachtende Wiederanstieg fällt zeitlich mit dem FFS-Rebound zusammen, signifikante Korrelationen zwischen beiden Parametern lassen sich jedoch nicht berechnen.

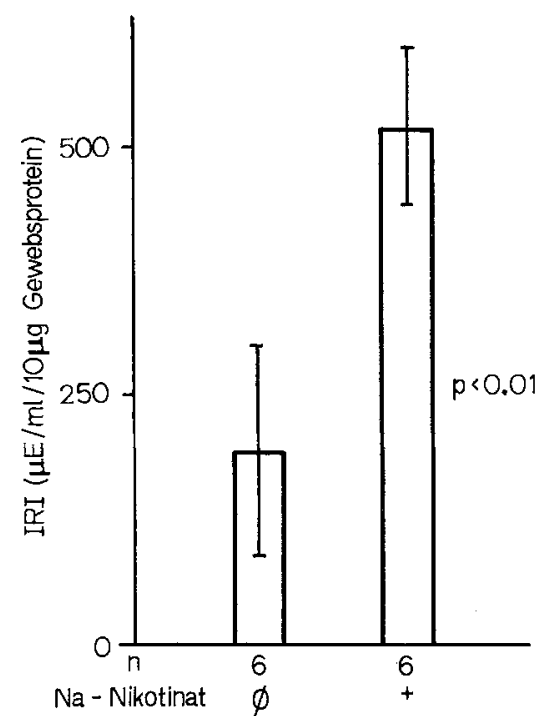

Abb. 2. Mittelwerte ( \pm S.E.M.) der Insulinsekretionsraten isolierter Langerhans'scher Inseln nach $120 \mathrm{~min}$ Incubation mit Glucose $(2 \mathrm{mg} / \mathrm{ml}$, linke Säule) und Glucose + Na-Nicotinat (1 $\mathrm{mMol}$, rechte Säule)

c) ILA (Tabelle 3). Die unterschiedliche quantitative Ausprägung des FFS-Rebound sowie das Plateau des FFS-Verlaufs zwischen der 90. und 180. min bei Stoffwechselgesunden lenkte das Interesse auf das Wirksamwerden des endogenen Insulins unter Nicotinsäureeinfluß. Daher wurde bei 7 orientierenden Belastungen die ILA zu den Zeiten $0,45,90$ und $150 \mathrm{~min}$ bestimmt. Wie aus Tabelle 3 hervorgeht, verursacht Nicotinsäure bei stoffwechselstabilen Diabetikern und Gesunden einen signifikanten Anstieg der ILA- Konzentration, deren Maximum 45 min nach Versuchsbeginn nachweisbar ist. Im Gegensatz dazu ist bei stoffwechsellabilen Diabetikern keine Wirkung zu konstatieren. Aus der Tabelle 3 geht weiter hervor, daß das Ausma $B$ des FFS-Rebound in negativer Korrelation $\mathrm{zu}$ der zum Zeitpunkt 45 min gemessenen absoluten ILAKonzentration steht, wie es sich durch Berechnung der Rangkorrelation nach Lienert (1962) $r=-0,92$ statistisch sichern läßt.

d) in vitro-Incubationsversuche mit Nicotinsäure (Abb. 2). Zur Bestätigung des in vivo gemessenen Einflusses der Nicotinsäure auf die Stimulation der Insulinsekretion wurden in 6 Paarvergleichen die Insulinfreisetzung isolierter Langerhans'scher Inseln bei
Incubation mit $1 \mathrm{mMol} \mathrm{Na}$-Nicotinat in Anwesenheit von Glucose gemessen. Aus Abb. 2 ist ersichtlich, daB Nicotinsäure einen signifikanten Anstieg der Insulinausschüttung hervorruft, der mehr als das Doppelte des unter Glucoseincubation allein beobachteten beträgt.

\section{Metabolische Differenzierung der Diabetesgruppen} (Abb. 3)

Die registrierten Unterschiede im Verhalten von Blutglucose-, FFS- und Glycerinkonzentrationen unter der Nicotinsäurebelastung zwischen stoffwechselstabilen und stoffwechsellabilen Diabetikern veranlaßte, nach weiteren Charakteristika für die metabolische Differenzierung beider Stoffwechseltypen zu suchen. Die Stimulation der endogenen ILA lenkte das Augenmerk auf die bei gleicher Kohlenhydratzufuhr zur Stoffwechselkompensation erforderliche exogene Insulindosis. Sie differiert bei den untersuchten Probanden zwischen dem stabilen und labilen Stoffwechseltyp signifikant um $22 \mathrm{E} /$ die. Somit wurden in das mathematische Modell der Differenzierung mit einbezogen:

1. Der Glucoseanstieg im Zeitraum $0-180 \mathrm{~min}\left(x_{1}\right)$

2. Die bei einer mittleren Kohlenhydratzufuhr von $300 \mathrm{~g}$ zur Stoffwechselkompensation erforderliche tägliche exogene Insulindosis $\left(x_{2}\right)$.

3. Der FFS-Anstieg im Zeitraum 45 bis $150 \mathrm{~min}\left(x_{3}\right)$.

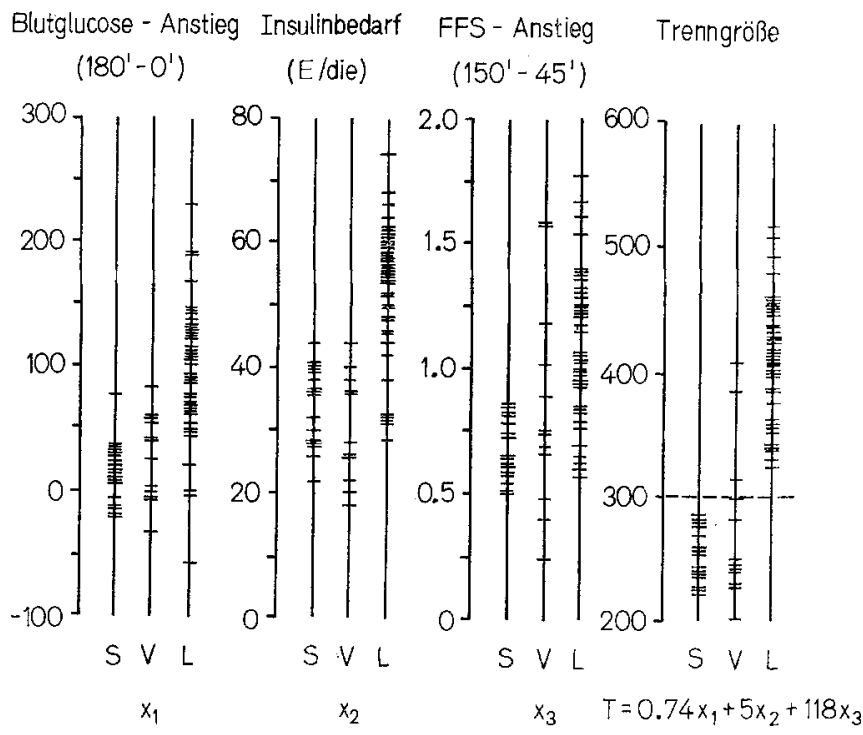

Abb. 3. Differenzierung des Stoffwechseltyps mittels der 30-minütigen Nicotinsäureinfusion nach der zwischen 0 und $180 \mathrm{~min}$ berechneten Blutglucosedifferenz $\left(x_{1}\right)$, dem mittleren exogenen Insulinbedarf $\left(x_{2}\right)$ und der zwischen 45 und $150 \mathrm{~min}$ errechneten FFS-Differenz $\left(x_{3}\right)$ mittels der durch Varianzanalyse aufgestellten Trennformel T. $(\mathrm{S}=$ stw.-stabiler D. $m$.; $V=$ stw.-stabiler D. m. mit vegetatativer Labilität; $\mathrm{L}=$ stw.-labiler D. m.)

Mit Hilfe der Diskriminanzanalyse (s. bei Weber 1964) wurde aus den in den Gruppen I und III erhaltenen Werten eine Trennformel errechnet. Sie geht aus 
der Lösung eines linearen Gleichungssystems hervor, dessen Koeffizienten die Korrelationen zwischen den Größen bzw. deren Standardabweichungen darstellen. Das Ergebnis lautet:

$$
T=0,74 \cdot x_{1}+5 \cdot x_{2}+118 \cdot x_{3}
$$

Die Abb. 3 demonstriert, daß keine der drei Einzelfaktoren eine sichere Trennung in den labilen und stabilen Stoffwechseltyp gestattet. Dagegen läßt sie sich aus den nach der Formel errechneten $T$-Werten durchführen. Die Schranke liegt bei einem $T$-Wert von 300 ; d.h., alle Diabetiker mit einem $T<300$ gehören dem stabilen, diejenigen mit einem $T>300$ dem labilen Stoffwechseltyp an. Die Gruppe der stabilen Diabetiker mit vegetativer Labilität nimmt - wenn man ihre Werte in die Trennformel einsetzt - eine Mittelstellung ein. Die überwiegende Mehrheit ist allerdings dem stabilen Stoffwechseltyp zuzurechnen.

\section{Diskussion}

Der Lipolyse-inhibierende Effect der Nicotinsäure, der sich in vivo im Absinken der FFS- und Glycerinkonzentrationen manifestiert, ist auf die rasche Akkumulation der Substanz im Fettgewebe zurückzuführen (Eaton 1963, Björntorp 1965, Carlson 1965, Schwandt et al. 1967). Nach Untersuchungen von Butcher et al. (1968), Schwandt u. Hartmann (1968) erfolgt die Lipolysehemmung über die Verminderung des zyklischen $3^{\prime}, 5^{\prime}$-AMP-Gewebsspiegels infolge Aktivierung der Phosphodiesterase (Krishna et al. 1966). Das sehr schnelle Abklingen des Effekts beruht auf der relativ kurzen Halbwertzeit. 30 bis 60 min nach oraler Applikation von 0,5 bzw. 1,0 g Nicotinsäure registrierten Gicoulhiac et al. (1962), Carlson et al. (1968) das Maximum der Nicotinsäurekonzentration im Blut, welcher mit der maximalen Depression der F'FS-Konzentration einherging. Dagegen ließen nach 3 bis 4 Std. gemessene Nicotinsäurekonzentrationen von 3,8 bis $1,0 \mu \mathrm{g} / \mathrm{l}$ den Lipolysehemmenden Effekt bereits vermissen. Daraus kann auf Grund der Konzentrationsveränderungen der FFS in unseren Versuchen gefolgert werden, daß die Nicotinsäure $60 \mathrm{~min}$ nach Infusionsende bereits eleminiert worden ist. Im Gegensatz zu den Fettstoffwechselparametern werden die Blutkonzentrationen der Glucose und deren Metaboliten durch Na-Nicotinat nicht signifikant beeinflußt (Pinter u. Pattee 1967, Carlson u. Östman 1965). Das schließt selbstverständlich die von Hepp et al. (1968) sowie Taskinen u. Nikkilä (1968) nachgewiesene Steigerung der Glucoseoxydation und $\mathrm{C}^{14}$-Inkorporation in Fettgewebe-TG aus $\mathrm{C}^{14}$-Glucose (Nikkilä 1968) unter Nikotinsäureeinfluß nicht aus.

Das rasche Sistieren der Nikotinsäurewirkung allein erklärt jedoch nicht den überschießenden FFS-Rebound, wie wir ihn übereinstimmend (mit Carlson et al. (1968), Gross u. Carlson (1968), Pinter u. Pattee (1967) nachweisen konnten. Er ist auf eine sekundäre Stimulation der Lipolyse zurückzuführen, wie es das Ver- halten der Serumglycerinkonzentration beweist. Ursächlich ist nach den tierexperimentellen Befunden von Pereira (1967) eine sekundäre Sekretionssteigerung des HVL-Adrenalsystems in Betracht zu ziehen. Dabei kommt den 11-OH-CS wahrscheinlich eine größere Bedeutung für die Induktion der Lipolysestimulation zu als den Katecholaminen (Pereira 1967). Die fehlenden Korrelationen zwischen FFS-Rebound und Konzentrationsanstieg der 11-OH-CS in unseren Untersuchungen sprechen nicht unbedingt gegen diese Annahme. Sie sind auf Grund der weitmaschig durchgeführten Parameterbestimmungen und der permissiven Rolle der Glucocorticoide bei der Lipolysestimulation (Fain 1968) kaum zu erwarten. Es scheint - wie wir auch bei der Lipolysehemmung mit 5-Methylpyrazol-3-Carbonsäure nachweisen konnten (unveröffentlichte Befunde) - eine geringe Steigerung der Glucocorticoidselsretion in der Lage zu sein, das lipolytische System im Fettgewebe für die Wirkung anderer Lipolyse-stimulierender Hormone zu sensibilisieren.

Das Ausmaß des FFS-Rebound nach der Nicotinsäureapplikation ist jedoch im wesentlichen abhängig von der Stimulierbarkeit der endogenen ILA. Wenn auch die orientierenden Untersuchungsergebnisse keine bindende Aussagekraft für das Gesamtkollektiv besitzen, so spricht einmal die positive Korrelation des exogenen Insulinbedarfs zur Höhe des FFS-Rebound für die Echtheit des Befundes. Zum anderen wird die Hypothese durch die Nicotinsäureinduzierte Stimulation der Insulinsekretion isolierter Langerhans'scher Inseln bestätigt. Wenn mit den ILA-Bestimmungen im fat pad auf Grund der allein durch Na-Nicotinat erzeugbaren Steigerung der Glucoseoxydation (Hepp et al. 1968), die die Grundlage der ILA-Messung darstellt, noch falsch positive Werte erhalten werden konnten, so ist das durch die radioimmunologischen Insulinbestimmungen bei den in vitro Untersuchungen ausgeschlossen. Letztere wurden in der Anordnung den in vivo beobachteten ILA-Veränderungen angepaßt, so daß neben der durch die Entspeicherung charakterisierten 1. Phase auch die metabolisch regulierte 2. Phase der Insulinsekretion miterfaßt wurde (Curry et al. 1968). Unsere in vitro Untersuchungsergebnisse stehen im Widerspruch zu denen von Malaisse et al. (1967), die an Rattenpankreasschnitten bei geringerer Glucosekonzentration und kürzerer Ineubationsdauer nur eine unbedeutende Steigerung der Insulinabgabe fanden. Ein direkter Vergleich der Ergebnisse ist nicht möglich, da unterschiedlich vorbehandeltes Tiermaterial und differente Methoden der IRI-Bestimmungen verwendet wurden.

Dagegen stehen unsere in vivo Ergebnisse in Einklang mit IRI-Messungen von Miettienen et al. (1968), die bei Stoffwechselgesunden unter kombinierten Glucase-Nicotinsäuregaben eine höhere Insulinausschüttungnachwiesen als unter alleiniger Glucoseapplikation.

Der Wirkungsmechanismus, der zur gesteigerten Insulinfreisetzung führt, ist unklar. Kausal ist an eine 
gesteigerte Bereitstellung des NAD aus Nicotinsäure (Bekierkunst u. Windman 1968) zu denken, welches die unmittelbare Muttersubstanz des NADP darstellt. NADP verursacht per se eine Stimulation der Insulinsekretion (Watkins u. Cooperstein 1968). Andererseits besteht die Möglichkeit, daß Nicotínsäure - ebenso wie in der Fettzelle (Hepp et al. 1968) - auch in der B-Zelle den Glucoseumsatz durch den Pentose-Phosphat-Shunt steigert, dessen Bedeutung für die Insulinsekretion Montague $u$. Taylor (1968) nachweisen konnten.

Zurückkommend auf die einleitende Fragestellung kann die metabolische Differenzierung zwischen labilem und stabilem Stoffwechseltyp mittels des Nicotinsäure-induzierten FFS-Rebound und des Blutglucoseverhaltens verifiziert werden. Die Abhängigkeit des Ausmaßes der sekundären Lipolysesteigerung von der Stimulierbarkeit des endogenen Insulins bzw. der insulinähnlichen Aktivität gestattet gewisse Rückschlüsse auf eine vorhandene Restaktivität der B-Zellen beim insulinbedürftigen Diabetes mellitus. Damit ist der juvenil-manifestierte Diabetes vom labilen Stoffwechseltyp durch das absolute endogene Insulindefizit (Paulsen et al. 1968, Parker et al. 1968) charakterisiert, während beim juvenilen Diabetes mit stabilem Stoffwechselverhalten - analog dem maturity-onset Diabetes (Floyd et al. 1968) - eine Stimulation der endogenen Insulinsekretion möglich ist.

\section{Literatur}

Anton, A.H., Sayre, D.F.: A study of the factors affecting aluminium oxide trihydroxyindole procedure for the analysis of catecholamines. J. Pharmacol exp. Ther. 138, 360-371 (1962).

Bekierkunst, A., Windman, I.: Nicotinic acid induced NAD synthesis in polymorphonuclear leucocytes. Life Sci. 7, $539-543$ (1968).

Björntorp, P.: The effect of nicotinic acid on adipose tissue metabolism in vitro. Metabolism 14, 836-839 (1965).

Bücher, Th., Czok, R., Lamprecht, W., Latzko, E.: Pyruvat. In: Methoden der enzymatischen Analyse, S. 253. Ed.: Bergmeyer, H.-U. Weinheim/Bergstr.: Verl. Chemie, 1962.

Butcher, R.W., Baird, Ch.E., Sutherland, E.W.: Effects of lipolytic and antilipolytic substances on adenosine 3', 5'-monophosphate levels in isolated fat cells. J. biol. Chem. 243, 1705-1712 (1968).

Carlson, L.A.: Inhibition of the mobilization of free fatty acids from adipose tissue. Ann. N.Y. Acad. Sci. 131, $119-142(1965)$.

- Orö, L.: The effect of nicotinic acid on the plasma free fatty acids. Demonstration of a metabolic type of sympathicolysis. Acta med. scand. 172, 641 - 645 (1962).

_- - Östman, J.: Effect of a single dose of nicotinic acid on plasma lipids in patients with hyperlipoproteinaemia. Acta med. scand. 183, 457-465 (1968).

- Östman, J. : Inhibition of the mobilization of free fatty acids from adipose tissue in diabetes. II. Effect of nicotine and acetylsalicylate on blood glucose in human diabetics. Acta med. scand. 178, $71-79$ (1965).

Curry, D.L., Bennett, L. L., Grodsky, G. M. : Dynamics of insulin secretion by the perfused rat pancreas. Endocrinology 83, 572-584 (1968).

Dolger, $H$. : Is juvenile diabetes always insulin deficient? On the significance of occasional success in treatment of diabetic children with oral therapy exclusively. 4 . Congr. I. D. F. Genf 1961, S. 356.

Duncombe, W.G.: The colorimetric micro-determination of non-esterified fatty acids in plasma. Clin. chim. Acta 9, 122-125 (1964).

Eaton, R.Ph.: In vitro inhibition of the fat-mobilizing action of nore-pinephrine. Proc. Soc. exp. Biol. (N.Y.) 114, 599-605 (1963)

Eggstein, M. : Eine neue Bestimmung der Neutralfette im Blutserum und Gewebe. II. Mitteilung: Zuverlässigkeit der Methode, andere Neutralfettbestimmungen, Normalwerte für 'Triglyzeride und Glyzerin im menschlichen Blut. Klin. Wschr. 44, 267-273 (1966 b).

Eggstein, M., Kreutz, F.H.: Eine neue Bestimmung der Neutralfette im Blutserum und Gewebe. I. Mitteilung: Prinzip, Durchführung und Besprechung der Methode. Klin. Wschr. 44, 262-267 (1966a)

Fain, J.N.: Effect of dibutyryl-3', 5'-AMP, theophylline and norepinephrine on lipolytic action of growth hormone and glucocorticoid in white fat cells. Endocrinology 82, 825-830 (1968).

Fajans, S.S., Conn, J.W.: Prediabetes, subclinical diabetes and latent clinical diabetes: interpretation, diagnosis and treatment. In: On the nature and treatment of diabetes. Ed.: B.S. Leibel a. G.A. Wrenshall, Excerpta med. Found. N.Y. 1965, p. 641.

Floyd, J.C., Fajans, St.S., Conn, J.W., Tihiffault, Ch., Knopf, R.F., Guntsche, E. : Secretion of insulin induced by amino acids and glucose in diabetes mellitus. J. clin. Endocr. 28, 266-276 (1968).

Gepts, W.: Pathologic anatomy of the pancreas in juvenile diabetes mellitus. Diabetes 14, 619-633 (1965).

Ginoulhiac, E., Tenconi, L.T., Chiancone, F.M.: 3-pyridineacetic acid and nicotinic acid: Blood levels, urinary elimination and excretion of nicotinic acid derivatives in man. Nature 193, 948-949 (1962).

Greenwood, F.C., Hunter, W.M., Glover, J.S.: The preparation of ${ }^{131}$ I-labelled human growth hormone of high specific radioactivity. Biochem. J. 89, 114-123 (1963).

Gross, R.C., Carlson, L.A.: Metabolic effects of nicotinic acid in acute insulin deficiency in the rat. Diabetes $\mathbf{1 7}$, $353-361$ (1968)

Hales, C.N., Randle, P.J.: Immunoassay of insulin with insulin-antibody precipitate. Biochem. J. 88, 137-146 (1963).

Hepp, D., Challoner, D.R., Williams, R.H.: Studies on the action of insulin in isolated adipose tissue cells. J. biol. Chem. 243, 4020-4026 (1968).

Hohorst, H.J., Kreutz, F.H., Bücher, Th.: Über Metabolitgehalte und Metabolitkonzentrationen in der Leber der Ratte. Biochem. Z. 332, 18-46 (1959).

Hugget, A.St. G., Nixon, D.A.: Enzymic determination of blood glucose. Biochem. J. 66, 12 (1957).

Joslin, E.P., Root, A.F., White, P., Marble, A.: The treatment of diabetes mellitus, S. 296. Philadelphia: Lea u. Febiger, 1959.

Krainick, H. G.: Zur Physiologie und Pathologie des intermediären Fettstoffwechsels. I. Mitt.: Bestimmung der Ketonkörper in kleinen Blutmengen. Klin. Wschr. 17, $450-451(1938)$.

Krishna, G., Weiss, B., Davies, J.I., Hynie, S.: Mechanism of nicotinic acid inhibition of hormone-induced lipolysis. Fed. Proc. 25, 719-724 (1966).

Lienert, G.A.: Verteilungsfreie Methoden in der Biostatistik, S. 194. Weisenheim am Glan: Verl. Anton Hain 1962.

Lowry, O.H., Rosebrough, N.J., Farr, A.L., Randall, R. J.: Protein measurement with the folin phenol reagent. J. biol. Chem 193, 265-275 (1951).

Malaisse, W.J., Malaisse-Lague, F., Mayhew, D.: A possible role for the adenylcyclase system in insulin secretion. J. clin. Invest. 46, 1724-1734 (1967). 
Miettinen, T.A., Taskinen, M.-R., Pelkonen, R., Nikkilä, E.A.: Effect of nicotinic acid on glucose utilization and plasma insulin in human subjects. Diabetologia 4, 311 (1968).

Molnar, G.D.: Observations on the etiology and therapy of "brittle" diabetes. Canad. med. Ass. J. 90, 953-959 (1964)

Montague, W., Taylor, K.W.: Insulin secretion and the intracellular concentrations of glucose-6-phosphate and 6-phosphogluconate in isolated islets of Langerhans. Biochem. J. 109, 30 (1968).

Nielsen, E., Asfeldt, V.H.: Studies on the specifity of the fluorimetric determination of plasma corticosteroids ad modum De Moor and Steeno. Scand. J. clin. Lab. Invest. 20, 185 - 194 (1967).

Nikkilä, E.A. : Effect of nicotinic acid on incorporation of glucose-C ${ }^{14}$ into triglycerides. Diabetologia 4, 312 (1968).

Parker, M.L., Pildes, R.S., Chao, K.L., Cornblath, M., Kipnis, D.M.: Juvenile diabetes mellitus, a deficiency in insulin. Diabetes 17, 27-32 (1968).

Poulsen, J.E., Jörgensen, K.R., Brunsfeldt, K. Investigaciones acerca de la insulina plasmatica en sujetos normales, obesos y diabeticos. Diab. clin. Endocr. y Nutr. 3, 207-223 (1968).

Pereira, J.N.: The plasma free fatty acid rebound induced by nicotinic acid. J. Lipid Res. 8, 239-244 (1967).

Pildes, R.S., Cornblath, M.: Personal communication (1967), zitiert bei D. M. Kipnis: Insulin antagonism and diabetes mellitus. In: Diabetes. Ed.: J. Östman. Proc. 6. Congr. I. D. F. Stockholm 1967. Excerpta Med. Found., Amsterdam 1969, S. 257.

Pinter, E.J., Pattee, C.J.: Biphasic nature of blood glucose and free fatty acid changes following intravenous nicotinic acid in man. J. clin. Endocr. 27, 440-443 (1967).

Renold, A.E., Martin, D.B., Dagenais, Y.M., Steinke, J., Nickerson, R.I., Sheps, M. C., Lauris, V.: Measurement of small quantities of insulin-like-activity using fat adipose tissue. I. A proposed procedure. J. clin. Invest. 39, $1499-1510(1960)$.

Schwandt, P., Hartmann, Th., Karl, H.J.: Der Einfluß von Nicotinsäure und Propranolol auf die lipolytische Wirkung von Catecholaminen und Peptidhormonen in vitro. Z. ges. exp. Med. 143, 79-84 (1967).

- - Untersuchungen zur Wirkung der Nikotinsäure auf die stimulierte Lipolyse. Z. klin. Chem. u. klin. Biochem. 6, 497-498 (1968).

Somogyi, M.: Unstable "brittle" diabetes. 4. Congr. I. D. F. Genf 1961, S. 301 .

Taskinen, M.R., Nikkilä, E.A.: Insulin action in fat cells; effect of intra- and extra-cellular fatty acid concentration. Diabetologia 4, 314 (1968).

Vallance-Owen, J., Hurlock, B., Pease, N.W.: Plasma insulin activity in diabetes mellitus. Lancet 1955 II, $583-587$.

Watkins, D., Cooperstein, S.J.: Insulin secretion from toadfish islet tissue stimulated by pyridine nucleotides. Science 162, $283-284$ (1968).

Weber, E.: Grundriß der biologischen Statistik. 5. Auflage. Jena: Fischer 1964.

Woodyatt, R.T.: Diabetes mellitus. In: Textbook of medicine, S. 628 . Ed.: R. L. Cecil. Philadelphia: W.B. Saunders Company 1934.

OA Dr. med. D. Michaelis

Institut für Diabetes

,Gerhardt Katsch"

DDR-2201 Karlsburg, Krs. Greifswald 